\title{
Knowledge and Utilization of Intermittent Preventive Treatment (IPT) for Malaria Control Among Pregnant Women Attending Antenatal Clinics in the Sunyani West District of Ghana
}

\author{
Evans Atenkperiga Ayiisi \\ Winwild Information and Research Consult, Sunyani West, Ghana
}

Email address:

evansatenkperiga@gmail.com

\section{To cite this article:}

Evans Atenkperiga Ayiisi. Knowledge and Utilization of Intermittent Preventive Treatment (Ipt) for Malaria Control Among Pregnant Women Attending Antenatal Clinics in the Sunyani West District of Ghana. Science Journal of Public Health.

Vol. 5, No. 3, 2017, pp. 254-262. doi: 10.11648/j.sjph.20170503.24

Received: February 21, 2017; Accepted: March 6, 2017; Published: April 15, 2017

\begin{abstract}
For this study, the knowledge and utilization of Intermittent Preventive Treatment (IPT) for malaria control among pregnant women attending antenatal clinics in the Primary Health Care Centers in the Sunyani West District of Ghana was assessed. A cross-sectional descriptive survey was conducted to assess antenatal accessibility and practices of IPT, knowledge of pregnant women on IPT use, and the effectiveness of IPT towards the reduction of malaria in pregnancy. The researcher used both secondary and primary data to elicit information for the study. The tools for the primary data collection were structured questionnaire and focused group discussion. Data was collected in two health facilities in the Sunyani West. Data was analyzed using Statistical Package for Social Science (SPSS) version 20.0 and the results were presented using frequencies, graph, cross tabulation and level of association using Chi-square. The study shows that the knowledge and utilization of IPT among pregnant women in Sunyani West District were very low. Majority of the respondents (68.2\%) did not have knowledge on ITP use. Thus at 95\% confidence level, the proportion of those who did not have knowledge on IPT use and those who had was highly significant $(\mathrm{p} 1 \neq \mathrm{P} 2)$. The major challenges that were identified to suppress the effectiveness of IPT utilization from the health care provider point of view included inadequate Antenatal Clinic trained staff, low facility coverage and late ANC reporting of pregnant women. This study, therefore, recommends that healthcare providers in the district should intensify education on the use of Intermittent Preventive Treatment of malaria in pregnant women through community durbars, and radio and Television programs.
\end{abstract}

Keywords: Intermittent Preventive Treatment, Malaria Control, Antenatal Accessibility, Effectiveness, Knowledge of IPT

\section{Introduction}

\subsection{Background Information of the Study}

Annually, over thirty (30) million African women in malaria prone areas become pregnant and are at risk of infection with Plasmodium falciparum [1]. Pregnancies in women residing in malaria rife regions are associated with a high frequency of patent parasitemia and clinical malaria [2]. In Ghana, it is reported that the prevalence of the malaria parasite is between $60 \%$ and $72 \%$ among pregnant women [3].

Malaria during pregnancy causes up to 10,000 maternal deaths annually and contributes to high rates of maternal morbidity including fever and severe anaemia, especially in first time mothers [4]. Steketee, Nahlen, Parise and Menendez [5] indicate that malaria is the cause of Low Birth Weight (LBW) and placental parasitaemia and 75,000 to 200,000 infant deaths per annum, are attributable to malaria infection in pregnancy. Intermittent Preventive Treatment for prevention of malaria in pregnancy (IPTp) is a key component of malaria control strategy in Ghana and Sulfadoxine-Pyrimethamine (SP) is a preferable drug [6].

Intermittent Preventive Treatment (IPT) consists of administration of curative dose of an efficacious anti-malaria drug at least twice during second and third trimesters of pregnancy during routinely scheduled antenatal clinic visits 
regardless of whether the woman is infected or not [7]. The drug is administered under supervision during antenatal care visits. Sulphadoxine Pyrimethamine (SP) is the drug recommended for the IPT strategy [8]. IPT with Sulphadoxine Pyrimethamine (SP) has been shown to reduce the risk of maternal anaemia, placental parasitaemia and low birth weight [6]. However, the knowledge and utility of IPT among pregnant women in Ghana is still low. This leads to maternal deaths and low birth weight. This challenge cannot be allowed to go unnoticed since good health is not only a basic human need but also a fundamental human right and a prerequisite for economic growth.

This study assesses IPT use among pregnant women attending Primary Health Centers for antenatal services in Sunyani West District in the Brong Ahafo Region of Ghana. Their knowledge, attitude towards IPT use, compliance with IPT and factors influencing IPT use were determined. It is anticipated that findings from this study would provide valuable information to guide planning of programmes to improve the use of IPT.

\subsection{Statement of the Research Problem}

Intermittent Preventive Treatment for prevention of malaria in pregnancy (IPT) is a major element of Malaria Control Strategy in Africa. Notwithstanding the evidence of the effectiveness of IPT strategy using SP in reducing the adverse effects of malaria during pregnancy, the uptake and coverage in Ghana is low. This is a key contributory factor of maternal morbidity and mortality and the low birth weight among most pregnant women in Ghana. IPT started in the Sunyani Municipality in 2005 after the programme had been successfully implemented in Nkransa in 2003 [9]. Underneath is a table showing IPT coverage in the Brong Ahafo Region.

Table 1. Percentage coverage of IPTp.

\begin{tabular}{llll}
\hline YEAR & IPT1 & IPT2 & IPT3 \\
\hline 2013 & 49.1 & 33.9 & 22.7 \\
2014 & 64.2 & 47.6 & 33.1 \\
2015 & 73.3 & 59.9 & 43.5 \\
\hline
\end{tabular}

Source: [9]

It is observed from the table above that the national target for IPT2 of $60 \%$ for 2008 was not reached even as at 2015 whilst the global target of $80 \%$ IPT2 by 2010 was very far from achieving even as at 2015. If these figures are accurately representative of IPT program in the district, then there is the need to understand what influenced this achievement and what can be done to further improve IPT coverage. Secondly, it is observed that although there has been a general improvement of IPT coverage over the years, there are 'dropouts' of subsequent doses of IPT. The cause of this 'dropout' also needs to be identified. At least $90 \%$ of Ghana's pregnant women attend ANC once before delivery [10] yet in the Sunyani West District only $73.3 \%$ were able to receive at least one dose of IPT in 2015. The possible factors accounting for this reduction also need investigation.
Factors responsible for the above may include late first time reporting to the $\mathrm{ANC}$, unreliable $\mathrm{ANC}$ attendance and unawareness of the benefits of IPT as against the effects of malaria in pregnancy. Others include availability of alternative services in the district and inaccessibility, both financially and geographically to health facilities, and poor attitude of women. On the part of the service providers, inadequate knowledge of the IPT policy, inadequate training of the personnel, non-availability of SP, scarcity of safe drinking water for the taking of SP at the health facilities, non-compliance to the Directly Observed Treatment (DOT) by the health workers may be contributory factors. Inadequate number of staff, poor staff attitude, lack of policy document, guidelines and protocols to follow in the facilities and inadequate monitoring and supervision of IPT activities in the district may be other factors that may also influence the uptake [9].

The reason behind this study was to assess the use of IPT among pregnant women attending Primary Health Centers in the Sunyani West District and to determine factors that influence the uptake using Chiraa and Nsoatre as study points. These study points were selected based on the recommendation of the district health director, the district disease control officer and the information officer because statistics prove that these are the only Health Centres that receive massive ANC attendance in the district annually.

\subsection{Research Objectives}

The main objective of this study was to assess the use of IPT among pregnant women attending Primary Health Care Centers in Sunyani West District for antenatal services. The specific objectives of the study include:

a. To determine Antenatal accessibility and practice of IPT at the ANC by pregnant women in Sunyani West District.

b. To find out the knowledge of pregnant women on IPT use within Sunyani West District and how it influences the number of SP uptake.

c. To find out the effectiveness of IPT and facilities towards the reduction of malaria among pregnant women in Sunyani West District.

\subsection{Research Questions}

a. Do pregnant women really have access to IPT?

b. Do pregnant women have knowledge on the use of IPT and can this influence the number of SP receive by women in the Sunyani West District?

c. How effective is the drug (SP) and health facilities in malaria control among pregnant women?

\section{Literature Review}

\subsection{ANC Accessibility and Practice of IPT by Pregnant Women}

In spite of the availability and implementation of IPT 
strategy in various health facilities in Sub-Sahara Africa, it is estimated that less than five percent $(5 \%)$ of pregnant women have access to effective malaria interventions; this is worse in the rural areas [11]. It has been estimated from a survey in four African countries that less than $20 \%$ of women use a prophylactic regimen close to the WHO recommendations [12]. In a study carried out in Ibadan, Southwest Nigeria by Falade et al. [13], IPT-SP was found to be highly effective in preventing maternal and placental malaria among parturient women as well as in improving pregnancy outcomes such as delivery of bigger babies and lower prevalence of pre-term deliveries and maternal anaemia [13].

Up to now, only few studies have investigated factors affecting compliance to IPT in most African countries including Ghana. The identified barriers to IPT use are related to concerns about SP safety and understanding of the protocol among health care providers and the community [14]. In a study in Tanzania, Newman [15], found that majority of respondents linked low compliance with IPT to poor acceptance of SP because of perceived side effects associated with SP. Launiala and Honkasolo [16] also asserted that pregnant women throw away drugs after leaving the clinic. Some factors influencing compliance include late enrolment, periodic shortages of drugs and health workers' underperformances. Majority of the late first ANC attendants by pregnant women that is, those who first attended ANC in the third trimester of pregnancy, attributed it to the fact that they had no problems during the pregnancy. Other reasons given included lack of money for transportation to the ANC, long distance to the ANC. Few women also gave reasons such as wanting the pregnancy to show first, not being able to leave farm work, attending an herbalist or a spiritualist clinic or being seen by a TBA. Similarly, an exploratory study in Mukono district, Uganda by Mbonye, Neema and Magnussen [2] found that an initial step towards a review of the policy aimed at improving access and use of SP in pregnancy is currently low. The results showed that SP is perceived to be an effective drug that cures malaria quickly. However, there are negative perceptions related to its use in pregnancy. SP is believed to be strong and weakens pregnant women, causes abortions and foetal abnormalities.

\subsection{Knowledge on IPT Use}

Intermittent Preventive Treatment is based on the use of anti-malarial drugs given in treatment doses at predefined intervals after quickening to clear a presumed burden of parasites. All asymptomatic pregnant women receive regular doses of SP as an IPT during the second and third trimesters while mothers with signs and symptoms of malaria receive rapid treatment according to the national treatment guidelines. The IPT with SP is provided as part of a comprehensive antenatal package with other drugs like haematinics and anti-helminthics to control maternal anaemia that is highly prevalent during pregnancy in the country [3].

Study conducted in Ghana by Antwi [17] shows that the knowledge of the pregnant women on IPT was significantly associated with the number of doses of SP received. The pregnant woman's knowledge about the purpose of taking $\mathrm{SP}$ at the ANC, the number of doses of SP to be taken during pregnancy, the timing of taking the SP as well as the effects of malaria on the mother and the baby influenced women to return for subsequent doses of SP. Perhaps, if a larger proportion are aware of the total number of doses of SP to be received during pregnancy, this would have increased the percentage that received all the three doses. Nganda et al. [18] showed that in Tanzania, attendance at health education sessions at the Maternal Child Health $(\mathrm{MCH})$ clinic was the only determining factor for IPT-SP use among pregnant women. Although, there were no structured health education plans for IPT at the ANCs when they were visited, this did not greatly affect the health education talks that were given by the health workers as not less than $70 \%$ of the respondents received their knowledge about IPT from the staff of the ANC. This proposed that education about IPT went on somehow during the ANC visits and this helped to increase coverage.

Why IPT: IPT of malaria during pregnancy is based on the assumption that pregnant woman living in areas of high malaria transmission has malaria parasites in her blood or placenta, whether or not she has symptoms of malaria. Malaria infection in the mother, therefore, increases the risk of Spontaneous abortion, Stillbirth, Pre-term birth Low birth weight and maternal anaemia. These effects are caused by malaria parasites being present in the placenta. These parasites are at the placenta sites, impairing passage of nutrients and oxygen from passing from the mother to the foetus. The use of the anti-malarial drugs given in treatment doses clears the sites of these parasites, allowing the free passage of nutrients and oxygen to the foetus. The free movement of nutrients and oxygen enables the foetus to develop normally, reducing the chances that a foetus will suffer the effects of malaria. IPT is important because many pregnant women can have malaria parasites without symptoms [3]. IPT is given to all asymptomatic pregnant women who report at the antenatal clinic in the second or third trimester but more especially; those of low gravidity (i.e. primigravida and secundigravida), those infected with HIV, adolescents and youth (10- 24 years) and those with unexplained anaemia. The drug of choice for IPT in Ghana is Sulfadoxine-Pyrimethamine. It is important to achieve an uninterrupted supply of SP for IPT use. The institutional management and midwife should check stock levels frequently, estimate for stock and dispense SP using the general principles for stock management and control [3].

Dosage: Sulfadoxine-Pyrimethamine (SP) or Fansidar is given as single adult dose (3 tablets) at regularly scheduled antenatal care visits during the second and third trimesters. Up to a maximum of 3 doses is recommended by the Ministry of Health. The first dose is given after quickening or after 16 weeks. The second dose is given at least one month after the first dose and the third dose is given at least one month after the second dose. Sulphadoxine-Pyrimethamine should be given at the ANC clinic or at where there is 
supervision of a midwife/health worker through a directly observed treatment (DOT) method [19]

\subsection{Effectiveness of IPT and Facilities}

Accurate record keeping is necessary to adequately monitor the progress and effectiveness of the programme and evaluate impact of the intervention [20]. A healthcare facility should establish and maintain a record for every woman and newborn that receives care. Data should be quickly summarized and feedback given to those collecting the data and should be used locally. Three groups of indicators are proposed to monitor and control activities of malaria in pregnancy at antenatal care facilities. Process indicators are those that measure whether or not interventions that are known to be effective in reducing the adverse consequences of malaria during pregnancy are being implemented. Intermittent Preventive Treatment given to pregnant women at routine antenatal care visits has been shown to promote healthier pregnancies and yield benefits for both the mother and her developing fetus. IPT of malaria during pregnancy can significantly reduce the occurrence of low-birth-weight infants and maternal anemia. IPT in infants also may have a major role in malaria prevention at the public health level [20].

\section{Method}

\subsection{Profile of Study Area}

The Sunyani West District (SWD) is one of the twentyseven Districts of the Brong Ahafo Region of the Republic of Ghana. The District lies between latitude $7^{\circ} 19^{\prime} \mathrm{N}$ and $7^{\circ}$ $35^{\prime} \mathrm{N}$ and longitudes $2^{\circ} 08^{\prime} \mathrm{W}$ and $2^{\circ} 31^{\prime} \mathrm{W}$. It shares boundaries with Wenchi Municipality to the North East, Tain District to the North, Berekum and Dormaa East to the West, Sunyani Municipal to the South East and to the Eastern borders of the District are Tano North and Ofinso North District. Sunyani West District has total land mass of about 1,658.7 square kilometers. The climatic zone of Sunyani West District falls within the Wet Semi-Equatorial region and therefore has two rainy seasons in a year. The current District population is estimated at 11,1741 [21].

\subsection{Study Design and Type}

The study is a descriptive cross-sectional study conducted in two public health facilities in two out of five sub-districts within the SWD from February to May, 2016. The research included only nursing mothers with babies less than six (6) months who attended ANC during the pregnancy before delivery and ANC staff. The study is descriptive in nature. The study is descriptive because it describes the utilization and knowledge of IPT among pregnant women.

\subsection{Population}

The study population comprised all nursing mothers with babies less than six (6) months and above who attended antenatal care in primary health care centers in the SWD and ANC staff. The total Women in Fertility Age (WIFA) in the Sunyani West District which is 25,924.

\subsection{Sampling Technique and Sample Size}

To obtain the appropriate information for this research, the researcher used systematic sampling technique. In systematic sampling, individuals are chosen at regular intervals (e.g., every fourth person) from the sampling frame. By taking estimate of the average clinic attendance of the month prior to the study, a sampling interval of four (4) was determined for each PHC. The first mother to be interviewed was picked by balloting from the ANC appointment cards submitted to the healthcare provider at the ANC. The study population comprises all submissive nursing mothers with babies less than six (6) months who attended ANC in primary health care centers during the pregnancy at the selected health facilities. The minimum number of mothers required for the study was 200 comprising only residents of the SWD. Hundred (100) respondents were required from each health care center. Sven (7) healthcare care providers at each PHC were selected for focus group discussion (FGD). The sample was determined from the total Women in Fertility Age (WIFA) in the SWD which is 25,924 . The sample was then calculated as below:

$$
\begin{gathered}
\mathrm{n}=\mathrm{N} / 1+\mathrm{N}\left(\mathrm{a}^{2}\right) \\
\mathrm{n}=\frac{25,924}{1+25924(0.072)} \quad \mathrm{n}=200
\end{gathered}
$$

$\mathrm{n}=$ sample size $\mathrm{N}=$ sampling frame $\mathrm{a}=$ margin of error $1=$ constant

\subsection{Data Collection Methods}

Both primary and secondary data were used to conduct the study. Primary data was collected using intervieweradministered questionnaire and FGD, designed by the investigator and pre-tested prior to use. Questionnaires included well-structured open-ended and close-ended questions. The questionnaire was written in English and was translated by the investigator in the language the respondents understood better if the respondent did not understand English. The secondary data was obtained from Internet, Books, Manuals, Reports, News Papers, Journals and data from the health facilities. The purpose of the secondary data was to enable the researcher to explore theories and practices surrounding the IPT.

\subsection{Data Analyses}

Data was analysed using Statistical Package for Social Sciences (SPSS) version 20.0 computer software. Data was summarised using tables and charts with frequencies and percentages. Chi-square/ Fischer Exact tests and the logistic regression analysis were used to test the association between categorical variables. A probability value ( $p$-value) less than 0.05 was taken to be significant at a confidence 
level of $95 \%$.

\subsection{Limitation of the Study}

The researcher was faced with the challenge of mobilizing enough money to print and get resources on time. The facility based nature of the study excluded those mothers who did not patronise antenatal clinic. The inability of some of the respondents $(<1 \%)$ complete the questionnaire after they have gone midway may affect the final results. However, the study was limited to the SWD as a result of financial and time constraints.

\section{Results Presentation and Analysis}

\subsection{Accessibility and Practice of IPT at ANC}

The Table 2 illustrates that a large proportion of the late first ANC attendants (33.3\%), that is, those who first attended $\mathrm{ANC}$ in the third trimester of pregnancy, attributed it to the fact that they had no problems during the pregnancy. Other reasons given included not having money/NHIS for transportation to the ANC (18.2\%), long distance to the ANC $(21.2 \%)$, wanting the pregnancy to show first $(18.2 \%)$ and not being able to leave farm work $(9.1 \%)$.

Table 2. Factors affecting IPT Uptake.

\begin{tabular}{llll}
\hline Gestation $>$ 6 months before attending $\mathbf{1}^{\text {st }} \mathbf{A N C}$ & Frequency & Percentage & Cum Percent \\
\hline Did not have money /NHIS & 5 & 18.2 & 33.3 \\
Did not have problems during the pregnancy & 10 & 9.1 & 51.5 \\
I could not leave my farm work & 2 & 21.2 & 60.6 \\
Long distance to the ANC & 6 & 18.2 & 81.8 \\
Wanted pregnancy to show first & 5 & 100.0 \\
Total & 33 & 100.0 \\
\hline
\end{tabular}

\subsection{Antenatal Record Information}

The median gestational age at receiving first dose SP was 22 weeks (16-35 weeks), second dose was 32 weeks (20-36 weeks) and that for third dose SP was 34 weeks (24-40 weeks). The average number of visits that had been made to the ANC was 5 (Standard Deviation, $\mathrm{SD}=3.1$ ). The number of visits that had been made ranged from as low as 1 to as high as 15 times as recorded in the ANC records books. One hundred and twenty (120) pregnant women $(60 \%)$ made four (4) or more antenatal visits. The Table 3 shows the number of SP doses received as given by respondents with correlation to the records in their ANC card recordings.

Table 3. Doses of SP Received.

\begin{tabular}{lllll}
\hline \multirow{2}{*}{ Gestation at ANC booking (Trimester) } & By Respondents & From Records & \\
\cline { 2 - 5 } & Frequency & $\mathbf{\%}$ & Frequency & \% \\
\hline Respondents receiving 1 dose & 9 & 4.5 & 7 & 3.5 \\
Respondents receiving 2 dose & 112 & 56.0 & 56 & 28.0 \\
Respondents receiving 3 dose & 54 & 27.0 & 137 & 68.5 \\
More than Three & 6 & 3.0 & 0 & 0.0 \\
Can't remember & 19 & 9.5 & - & - \\
Total & 175 & 100 & 200 & 100 \\
\hline
\end{tabular}

The Table 3 indicates that $4.5 \%$ of the respondents received one (1) dose of SP whilst the records depicted $3.5 \%$. Respondents who had received SP two (2) were 112 (representing 56\%) whilst the records indicated 56 (representing 28\%). Others, 54 (representing 27\%) also received 3 doses of SP whilst records reflected 137 (representing 68.5\%). However, 19 (representing 9.5\%) of the respondents could not recall the number of doses taken and 6 (representing 3\%) said they received more than three times

\subsection{Level of Knowledge on IPT}

Table 4 summarised the knowledge of the respondents on IPT use whereas table 5 depicts the associations between the knowledge of the respondents about IPT and the number of SP doses received (coverage of IPT) using Chi-square tests.

Table 4. Knowledge of Respondents on IPT,

\begin{tabular}{|c|c|c|c|c|c|}
\hline \multirow[t]{2}{*}{ Component of knowledge in IPT } & \multicolumn{2}{|c|}{ Respondents knowing right answer } & \multicolumn{2}{|c|}{ Respondents not knowing right answer } & \multirow[t]{2}{*}{ Difference B/N 2 proportions } \\
\hline & Freq. (P2) & $\%$ & Freq. (P1) & $\%$ & \\
\hline Purpose of SP & 87 & 43.7 & 113 & 56.3 & $0.03<\mathrm{P} 1-\mathrm{P} 2<0.04$ \\
\hline No. of doses SP during pregnancy. & 64 & 32.0 & 136 & 68.0 & $0.27<\mathrm{P} 1-\mathrm{P} 2<0.45$ \\
\hline Interval of taking SP & 153 & 76.5 & 47 & 23.5 & $-0.62<\mathrm{P} 1-\mathrm{P} 2<-0.45$ \\
\hline Malaria effect in pregnancy & 84 & 42.0 & 116 & 58.0 & $0.06<\mathrm{P} 1-\mathrm{P} 2<0.26$ \\
\hline Mal. effects on unborn baby & 80 & 40.0 & 120 & 60.0 & $0.10<\mathrm{P} 1-\mathrm{P} 2<0.30$ \\
\hline
\end{tabular}

Table 4 shows that there is a strong evidence that the proportion between those who do not have knowledge on IPT and those who have is highly significant since zero (0) is not contained in the interval in testing the difference between the two proportions thus, $\mathrm{P} 1-\mathrm{P} 2 \neq 0$. This means that at $95 \%$ confidence interval, the knowledge level of those who do not have knowledge on IPT use and those who have is highly significant. Hence $\mathrm{P} 1>\mathrm{P} 2$ 
Table 5. Comparison of Knowledge of Women on IPT and the Number of SP received.

\begin{tabular}{llllllll}
\hline Component of knowledge in IPT & \multicolumn{2}{l}{ Respondent knowing the right answer } & \multicolumn{2}{l}{ Number of SP doses } & & \\
\hline & Freq. & $\mathbf{\%}$ & $\mathbf{1 ~ n \%}$ & $\mathbf{2 ~ n \%}$ & P-Value & $\mathbf{3 ~ n \%}$ & P-Value \\
\hline Purpose of SP & 87 & 43.7 & $6(100)$ & $36(69.2)$ & 0.30 & $70(50.0)$ & 0.04 \\
No. of doses SP during pregnancy & 64 & 32.0 & $4(66.7)$ & $14(26.9)$ & 0.40 & $46(32.6)$ & 0.02 \\
Interval of taking SP & 153 & 76.5 & $4(66.7)$ & $33(63.5)$ & 0.35 & $116(82.3)$ & 0.722 \\
Mal. effects on pregnant Woman & 84 & 42.0 & $1(16.7)$ & $29(55.0)$ & 0.35 & $54(62.4)$ & 0.733 \\
Mal. effects on baby & 80 & 40.0 & $3(50.0)$ & $33(63.5)$ & 0.574 & $44(31.2)$ & 0.831 \\
\hline
\end{tabular}

P-value 1 shows the level of association between the knowledge variable and the respondent receiving a second dose of SP after a first dose had been received and P-value 2 shows the association between the knowledge variable and the respondent receiving a third dose after a second dose of SP had been received.

\subsection{Effectiveness of IPT and Facilities}

Table 6 and 7 illustrates two interventions adopted by pregnant women for the prevention of malaria especially during pregnancy. The two malaria interventions include the use of Insecticide Treated-Bed Net (ITN) and the used of SP. These two interventions are assessed to determine the effectiveness of malaria interventions practiced by pregnant women.

Table 6. ITN use among Pregnant Women.

\begin{tabular}{llllllll}
\hline \multicolumn{2}{ll}{ Respondents owning ITN } & \multicolumn{5}{l}{ Respondents sleeping under net previous night } \\
\hline Yes & No & \multicolumn{5}{l}{ Yes } & No \\
\hline $\mathbf{N}$ & $\mathbf{\%}$ & $\mathbf{N}$ & $\mathbf{\%}$ & $\mathbf{N}$ & $\mathbf{\%}$ & $\mathbf{N}$ & $\mathbf{\%}$ \\
\hline 137 & $(68.5)$ & 63 & $(31.5)$ & 129 & $(64.5)$ & 71 & $(35.5)$ \\
\hline
\end{tabular}

Table 7. SP Received 2015.

\begin{tabular}{llllll}
\hline 1st & 2nd & 3rd & Total ANC attendance & IPT trained Staff & Total ANC staff \\
\hline 1,174 & 1,083 & 670 & 4950 & 2 & 9 \\
\hline
\end{tabular}

From Table 6 and 7, to measure whether or not interventions are known to be effective in reducing the adverse consequences of malaria during pregnancy are being implemented, using the three (3) groups of indicators proposed to monitor effectiveness or control activities of malaria in pregnancy at antenatal care facilities, the following are the findings as calculated from the tables above. Process indicators that measure whether or not interventions are known to be effective in reducing the adverse consequences of malaria during pregnancy are being implemented.

$-\%$ of antenatal clinic staff trained to deliver IPT to pregnant women

$$
\frac{\text { Number } \text { of ANC staff trained to deliver IPT }}{\text { Number of ANC staff }} \times 100
$$

$=\frac{3 \times 100}{9}=33 \%$

$-\%$ of Health facility implementing IPT

$\frac{\text { Number of Health Facilities Implementing IPT }}{\text { Number of Health Facilities in District }} \times 100$

$=\frac{5 \times 100}{9}=55.5 \%$

Outcome indicators: These indicators should reflect the change in knowledge and practices

$-\%$ of pregnant women receiving IPT1
Number of pregnant women who receive IPT1 $\times 100$

Total Number of Attendants (New Registrants + Continuing)

$=\frac{1174 \times 100}{4950}=23.7 \%$

-\% of pregnant women receiving IPT2

$\frac{\text { Number of pregnant women who receive IPT2 }}{\text { Number of Attendants (Registrants }+ \text { Continuing) }} \times 100$

$=\frac{1083 \times 100}{4950}=21.9 \%$

$-\%$ of pregnant women receiving IPT3

Number of pregnant women who receive IPT3

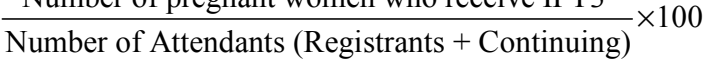

$=\frac{670 \times 100}{4950}=13.5 \%$

$-\%$ of pregnant women who report that they slept under an ITN the previous night.

$\frac{\text { Number of pregnant who reported sleeping under ITN the previous night }}{\text { Number of Attendants (Registrants }+ \text { Continuing) }} \times 100$

$$
=\frac{129 \times 100}{200}=64.5 \%
$$

From Figure 1, the records obtained from the $995.5 \%$ of the nursing mothers did not suffer any birth defects such as still birth, low birth weight and spontaneous abortion. It turned out that $87.4 \%$ of the mothers did not experience malaria during pregnancy and $94.4 \%$ of the mothers did not 
also experience anaemia during the pregnancy.

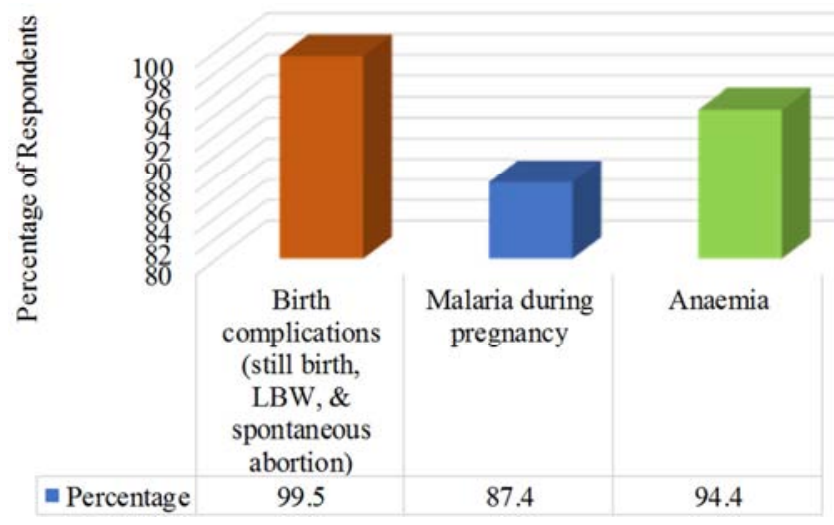

Figure 1. Effectiveness of SP in the Control of Malaria.

\section{Discussion}

\subsection{Antenatal Accessibility and Practice of IPT by Pregnant Women}

The President Malaria Initiative [10] indicated that SPT1 should be given after quickening or after 16 weeks. However, the present study revealed that large proportion of the late ANC attendants, that is, those who first attended ANC in their third trimester of pregnancy, attributed it to the fact that they had no problems during the pregnancy. Some other reasons given included lack of money or health insurance, long distance to the ANC, wanting the pregnancy to show first and not being able to leave farm work. These findings are similar to Antwi, [18] who also found majority of the late first ANC attendants to have attributed it to the fact that they had no problems during the pregnancy, no money for transportation, long distance to the ANC, and inability to abandon farm work for the health facility.

The median gestational age at receiving first dose of SP was 22 weeks (16-35 weeks), second dose was 32 weeks (2036 weeks) and that for third dose SP was 34 weeks (24-40 weeks). The average number of visits that had been made to the ANC was 5 (Standard Deviation, $\mathrm{SD}=3.1$ ). The number of visits that had been made ranged from 1 to 15 times as recorded in the ANC records books. One hundred and twenty (120) pregnant women $(60 \%)$ made four (4) or more antenatal visits. In Table 3, the number of SP doses received as given by respondents with reference to the records in their ANC card recordings showed several discrepancies. It revealed that $4.5 \%$ of the respondents admitted that they received one (1) dose of SP whilst records showed that 3.5\% received one (1) dose of SP. Respondents who had received SP two (2) as given by themselves were $56 \%$ whilst records indicated 28\%. Also, 27\% said to have received three (3) doses of SP whilst the card records reflected $68.5 \%$. These variations may be attributed to factors such as poor documentation on the part of the health workers or clients' inability to remember vividly the number of SP doses received.

During the FGD, the health workers also reported that majority of the pregnant women also threw the drugs away after taken the drugs outside the health facility. This means some facilities do not practice the DOT policy as recommended for every facility. This DOT policy recommends three doses of SP to be administered to HIVnegative pregnant women starting after quickening (16 weeks or thereafter) with each dose administered monthly [3]. The last dose of SP should be administered at least one month before delivery. According to GFATM [3], all doses are to be administered under direct observation. HIV-positive pregnant women are expected to receive monthly doses of SP after quickening (with a total of four doses) except if they are receiving cotrimoxazole. According to $\mathrm{MoH}$ [19], Sulphadoxine-Pyrimethamine should be given at where there is supervision by a midwife/health worker through a directly observed treatment (DOT) method. The panelists of the FGP also indicated that in a situation where drugs are taken under the supervision of staff, some pregnant women still hide the Tablets under their tongues and spit them out immediately they stepped outside the room. These findings support Launiala et al. [16] who asserted that pregnant women threw away drugs after leaving the clinic. This means research question one is answered; as large proportion of the late ANC attendants in the third trimester of pregnancy, attributed it to the fact that they had no problems during the pregnancy and besides, some pregnant women did not see the need in taken the drug (SP) hence spiting or throwing the drugs away.

\subsection{Knowledge of Pregnant Women on IPT Use and Its Influence on SP Uptake}

According to GFATM [3], all asymptomatic pregnant women receive regular doses of SulphadoxinePyrimethamine (SP) as an Intermittent Preventive Treatment (IPT) during the second and third trimesters, while mothers with signs and symptoms of malaria get prompt treatment according to the national treatment guidelines. In Table 4, there is strong evidence that the proportion between those who did not have knowledge on IPT and those who have knowledge on IPT is highly significant since zero (0) is not contained in the interval in testing the difference between the two proportions $(\mathrm{P} 1-\mathrm{P} 2 \neq 0)$. This means that at $95 \%$ confidence interval, the knowledge level of those who did not have knowledge on IPT use and those who had was highly significant. Majority (53.3\%) of the women did not know the purpose of SP and $68 \%$ did not know the number of doses to be taken during pregnancy. This may be detrimental to pregnant women because HIV-negative pregnant women are likely to take more or less than the required three (3) doses if there is poor documentation and forgetfulness on the part of the health care provider.

The pregnant woman knowing the purpose of taking SP and the number of doses of SP to be taken, positively influenced her coming back to take a third dose of SP after a second dose had been taken $(p=0.04, p=0.02)$ whilst these did not influence her taking a second dose after having received the first dose $(\mathrm{p}=0.30, \mathrm{p}=0.40)$. Knowledge of the number of doses of SP that needed to be taken, the interval of taking the 
SP, effects of malaria on the mother and her baby, influenced her coming back for a third dose of SP after the second dose had been received $(\mathrm{p}=0.001)$ but not her coming for a second dose after the first dose had been received. Again, the majority $(85.5 \%)$ of the respondents affirmed that the health staff were their major source of knowledge about IPT while few $(8.5 \%)$ women mentioned the media (television and radio) and the remaining (6\%) women mentioned their fellow women as their source of knowledge. Similarly, Nganda et al. [18] found that in Tanzania, attendance at health education sessions at the $\mathrm{MCH}$ clinic was the only determining factor for IPTp-SP use among pregnant women. The findings mean research question two is answered; as pregnant women have little knowledge on IPT use and besides, the pregnant woman knowing the purpose of taking SP and the number of doses of SP to be taken, has the tendency of positively influencing her coming back to take a third dose of SP after a second dose had been taken.

\subsection{Effectiveness of IPT Towards the Reduction of Malaria Among Pregnant Women}

The President Malaria Initiative [10] indicated that to measure whether or not interventions are known to be effective in reducing the adverse consequences of malaria during pregnancy, three (3) groups of indicators are proposed to monitor the effectiveness or control activities of malaria in pregnancy at the ANC. These indicators include Process indicators, Outcome indicators and impact indicators. However, only Process indicators and Outcome indicators were employed in this study. In measuring whether or not the IPT interventions are effective in reducing the adverse consequences of malaria during pregnancy, the percentage of antenatal clinic staff trained to render IPT services to pregnant women was $33 \%$ which is very low as compared to the Ministry of Health requirement given by the President Malaria Initiative [10]. Also, the percentage of Health facilities implementing IPT in the Sunyani West is moderate (55.5\%). These had accoutered for low uptake of IPT, and inaccessibility thereby making IPT use and facilities ineffective.

The outcome indicators should reflect the change in knowledge and practices using the percentage of pregnant women receiving IPT1, IPT2 and IPT3 as recommended by the President Malaria Initiative in 2009. Thus, the percentages of women receiving IPT1, IPT2 and IPT3 in 2015 were $23.7 \%, 21.9 \%$ and $13.5 \%$ respectively. This decrease in number from IPT1 to IPT3 were attributed to certain factors that were given by ANC staff during the focused group discussion which include; late ANC reporting by pregnant women at their first visit and low level of women on the importance of IPTp. The findings of the present study are in line with the Global Fund Training Manual [3] that indicated that the antenatal period is a critical time to reach women with effective malaria prevention and treatment messages, services, and products. Most women in Ghana make at least one antenatal visit to a health facility, although a majority present late in the pregnancy.
In terms of effectiveness of the SP, the study revealed that the drug has achieved $99.5 \%$ as far as low birth weight of baby, still birth and spontaneous abortion are concerned. In terms of malaria during pregnancy $87.4 \%$ and anaemia $94.4 \%$. The findings mean SP is effective in the prevention of malaria and anaemia during pregnancy as well as some birth complications such as low birth weight of baby; still birth and spontaneous abortion. The findings correspond with Marielle et al. [6] who also found that IPT given to pregnant women at routine antenatal care visits has been shown to promote healthier pregnancies and yield benefits for both the mother and her developing foetus. IPT of malaria during pregnancy can significantly reduce the occurrence of lowbirth-weight infants and maternal anemia. IPT in infants also may have a major role in malaria prevention at the public health level [6]. This means research question three is answered; as the effectiveness of health facility in the utility of the IPT is ineffective due to inadequate staff and late reporting of pregnant women. However, the SP seems to be effective towards malaria reduction during pregnancy.

\section{Conclusion}

Large proportion $(33.3 \%)$ of the late first ANC attendants, attended ANC in the third trimester of pregnancy which is attributed to the fact that they had no problems during the pregnancy. Besides, some pregnant women did not see the need to take the drug (SP) hence spiting or throwing the drugs away.

The study shows that the knowledge and the utilization of IPT among pregnant women in the Sunyani West District were very low. It was also realized that majority $(68.2 \%)$ of the pregnant women did not have knowledge on IPT use while $31.8 \%$ had knowledge on IPT use. Thus, at $95 \%$ confidence level, the proportion of those who did not have knowledge on IPT use and those who had, was highly significant $(\mathrm{P} 1>\mathrm{p} 2)$. Besides, the pregnant woman knowing the purpose of taking SP and the number of doses of SP to be taken, positively influenced her coming back to take the third dose of SP after a second dose had been taken $(\mathrm{p}=0.04$, $\mathrm{p}=0.02$ ) whilst these did not influence her taking a second dose after having received the first dose $(p=0.30, p=0.40)$.

The effectiveness of health facility in the utility of the IPT is ineffective due to inadequate staff and late reporting of pregnant women. However, the SP seems to be effective towards malaria reduction during pregnancy. The percentage of antenatal clinic staff trained to deliver IPT to pregnant women was $33 \%$, the percentage of health facilities implementing IPT was 55\%, pregnant women receiving IPT2 was $21.9 \%$, pregnant women receiving IPT3 was $13.5 \%$ and percentage of pregnant women who report that they slept under an ITN the previous night.

$64.5 \%$. This means that the IPT facilities in the SWD were ineffective.

The plausible interventions by the Ministry of Health to address the gaps and deficiencies in IPTp include developing a health promotion package to explain the benefits of SP as 
IPT pageant and to counteract the wrong perception that SP could harm both mother and child. In addition, health workers should be provided continuous education and training to improve their knowledge about malaria during pregnancy and in particular IPTp strategy and DOT scheme. Activities of health workers manning the antenatal clinics should also be supervised.

\section{References}

[1] Marchesini, T., \& Crawley, J. (2004). Malaria, iron deficiency and anaemia control. An Update of Roll Back Malaria. Geneva: WHO.

[2] Mbonye, A. K., Neema, S., \& Magnussen, P. (2005). Perceptions on use of sulfadoxine- pyrimethamine in pregnancy and the policy implications for malaria control in Uganda. Journal of Health Policy, 2, 6.

[3] Global Fund Training Manual. (2005). Intermittent Preventive treatment of Malaria in Pregnancy. Training Manual for Health Providers. Participants guide.

[4] Savage, E., Msyamboza, K., Gies, S., \& D'Alessandro, H. (2007). Indicator for monitoring malaria control in pregnancy in sub-Saharan Africa. BJOG, 114, 1222-1231.

[5] Steketee, R., Nahlen, B., Parise, M., \& Menendez, C. (2001). The burden of malaria in pregnancy in malaria-endemic areas. American Journal of Tropical Medicine and Hygiene, 64, 2835 .

[6] Marielle, K. B. A., Denisa, E. I. C., Modeste, M. M., Eric, K., Pierre, B. M., Elie, M., \& Maryvome, K. (2003). Prevalence of Plasmodium Falciparum Infection in Pregnant women in Gabon. Malaria Journal, 2, 1-17.

[7] Peter, P. J., Thigpen, M. C., Parisen, M. E., \& Newman, R. D. (2007). Safety and toxicity of Sulfadoxine pyrimethamine implications for malaria prevention in pregnancy using.

[8] WHO. (2004). A strategic framework for malaria prevention and control during pregnancy in the Africa Region. RBM Malaria in Pregnancy Info Sheet 2005. Geneva: World Health Organization.

[9] Ghana Health Service Annual Report, 2015.

[10] President Malaria Initiative. (2009). Malaria Operational
Plan, Year Two (FY09). Ghana.

[11] WHO/RBM. (2005). Children and Malaria. Roll Back Malaria Department. Geneva: WHO.

[12] Enato, E. F., Okhamafe, A. O., \& Okpere, E. E. (2007). A survey of knowledge, attitude and practice of malaria management among pregnant women from two health care facilities in Nigeria. In Acta Obstetric Gynecological Scand., 86 (1), 33-6.

[13] Falade, C. O., Yusuf, B. O., Fadero, F. F., Mokolu, O. A., Hamer, D. H., \& Salako, L. A. (2007). Intermittent preventive treatment with Sulfadoxine-Syrimethamine is effective in preventing maternal and placental malaria in Ibadan, southwestern Nigeria. Malaria Journal, 6, 8 .

[14] Mubyazi, A., Bloch, P., Kamugisha, M., Kituua, A., \& Ijimba, J. (2005). Intermittent preventive treatment of malaria during pregnancy: A qualitative study of knowledge, attitudes and practices of district health managers, antenatal care staff and pregnant women in Korogwe district, Northern eastern Tanzania. Malaria Journal, 4, 31.

[15] Newman, R. D. (2006). Malaria prevention during pregnancy; assessing burden one year after implementing a program of 2006: the disease preventive treatment in Koupela district, Burkina Faso. American Journal of Tropical Medical and Hygiene, 75, 205-211.

[16] Launiala, A., \& Honkasolo, C. J. (2007). Reduction in mortality and morbidity from malaria in Gambian children. Lancet, 345, 479-483.

[17] Antwi, G. D. (2010). Factors influencing the uptake of Intermittent Preventive Treatment of Malaria in Pregnany in the Bosomtwi District of Ghana. French Embassy Small Grants programme in the Humanities and Social Sciences, 5254. Ghana: Accra.

[18] Nganda, R. Y., Drakeley, C., Reyburn, H., \& Marchant, T. (2004). Knowledge of malaria influences the use of insecticide treated nets but not intermittent presumptive treatment by pregnant women in Tanzania. Malaria Journal, 12 (3), 42.

[19] Ministry of Health, Ghana. (2009). Malaria Action Plan: 19931997. Ghana: Accra, (unpublished).

[20] Roll Back Malaria, RBM. (2011). Children and Malaria. Roll Back Malaria Department. Geneva: WHO.

[21] Ghana Statistical Service. (2012). Municipal Population and Housing Census Unpublished. Ghana: Sunyani. 